\title{
Computer Simulation: the imaginary friend of auxin transport biology
}

Philip Garnett ${ }^{1,4}$, Arno Steinacher ${ }^{3,5}$, Susan Stepney ${ }^{2}$, Richard Clayton ${ }^{3}$ and Ottoline Leyser ${ }^{1}$.

1. Department of Biology, University of York, PO Box 373, YO10 5YW.

2. Department of Computer Science, University of York, YO10 5DD.

3. Department of Computer Science, University of Sheffield, S1 4DP.

4. prg500@york.ac.uk

5. a.steinacher@sheffield.ac.uk

\section{Abstract}

Regulated transport of the plant hormone auxin is central to many aspects of plant development. Directional transport, mediated by membrane transporters, produces patterns of auxin distribution in tissues that trigger developmental processes, such as vascular patterning or leaf formation. Experimentation has produced a lot of largely qualitative data providing strong evidence for multiple feedback systems between auxin and its transport. However the exact mechanisms concerned remain elusive and the experiments required to evaluate alternative hypotheses are challenging. Because of this, computational modelling now plays an important role in auxin transport research. Here we review current approaches and underlying assumptions of computational auxin transport models, including recent attempts to unify conflicting mechanistic explanations. In addition we discuss in general how computer simulation of these processes is proving to be an increasingly effective method of hypothesis generation and testing, and how simulation can be used to direct future experiments.

\section{Introduction}

Auxin was one of the first plant hormones to be discovered, with evidence for its existence provided 130 years ago by Charles and Francis Darwin ${ }^{(1)}$. Despite its long history, understanding its functions still presents many challenges to plant science. Auxin is involved in diverse aspects of plant patterning and development. These include the establishment of the embryonic axes ${ }^{(2-4)}$, the patterning of the root meristem ${ }^{(5-6)}$, the regulation of lateral root initiation $^{(7)}$, phyllotaxis - the positioning of leaf and flower organ primordia ${ }^{(8-9)}$, the regulation of shoot branching ${ }^{(10-12)}$, and the development of vascular tissue ${ }^{(13-14)}$. All of these processes involve dynamic patterns of auxin distribution and redistribution across tissues. There are still no tools available to make auxin gradients directly visible in living tissues. However, auxin-induced transcription can be visualised using auxin responsive promoter-reporter fusion constructs such as DR5:GFP, a synthetic auxin-inducible promoter fused to Green Fluorescent Protein (GFP). Using such reporters, gradients of auxin response can be correlated with pattern formation and cell differentiation, and dynamic redistribution of auxin during development can be inferred. The importance of auxin distribution patterns, and their dynamic nature, has in turn focused attention on the underlying process of regulated transport of auxin. Because of the difficulties of observing auxin transport mediated patterning directly in plants mathematical and computational models have emerged as powerful tools for generating and testing hypotheses. In this review we will focus on simulations modelling the cellular mechanisms proposed to be responsible for auxin transport and its directionality. Our emphasis is on the utility of these models in contributing 
to biological understanding, and thus our discussion is a qualitative one, centred on the core assumptions of the models. For a more mathematically explicit discussion we recommend Krupinski and Jonsson ${ }^{(15)}$.

\section{Auxin}

The most abundant natural auxin is indole-3-acetic acid (IAA). At the low pH of the extracellular space (the apoplast) a fraction of the auxin is protonated, and thus able to cross the plasma membrane and enter cells passively. However, the cytoplasmic $\mathrm{pH}$ is much higher, so once inside the cell auxin is almost entirely ionised and thus unable to leave without active protein-mediated transport (Fig. 1). This phenomenon is known as the acid trap or the chemiosmotic theory and is summarised by Rubery \& Sheldrake ${ }^{(16)}$ and Raven $^{(17)}$.

Export of auxin from cells relies on membrane localised efflux proteins (Fig. 1). There are several protein families that have been shown to act in auxin efflux. Prominent among these are members of the ABCB transporter family and members of the PIN family ${ }^{(2,18-22)}$. A great deal of attention has focused on the PIN family because PIN proteins are often found distributed asymmetrically around the membrane of a cell. This asymmetric localisation of PIN transporters is important because it enables directional auxin transport; diverse experiments support the hypothesis that the direction of auxin transport across tissues is determined by the co-ordinated positioning of PIN proteins ${ }^{(23)}$. Auxin can also be actively transported into cells via members of the AUX/LAX influx protein family ${ }^{(24)}$.

\section{Auxin's Roles in Plant Development}

During the early stages of embryogenesis, the apical-basal axis is defined by dynamic patterns of auxin accumulation, with auxin accumulating first at the shoot pole and then later at the root pole. Auxin accumulation at these sites is required to establish the shoot apical meristem (SAM) at the shoot pole, and the root apical meristem at the root pole ${ }^{(3)}$. During post-embryonic development these small groups of stem cells are responsible for the production of the shoot and root systems respectively. This reversal of auxin distribution in the apical/basal axis during embryogenesis is correlated with the redistribution of PIN proteins from the apical face to the basal face of embryo cells ${ }^{(2,25)}$. The importance of these events is demonstrated by the fact that the establishment of the embryonic axis is disrupted in pin mutants, and in wild-type plants to which pharmacological inhibitors of auxin transport have been applied.

After germination the SAM goes on to initiate the successive organs of the shoot. During this process, known as phyllotactic patterning, auxin directs the positioning of primordia for lateral organs, such as leaves, in a regular pattern around the shoot. This process has long fascinated plant scientists and mathematicians because of the geometry of the patterns produced. One common pattern is spiral phyllotaxis, in which organs are produced in a spiral around the shoot axis separated by approximately 137.5 degrees, the golden angle derived from the ratio of consecutive numbers in the Fibonacci sequence. During phyllotactic patterning, PIN protein localisation in cells of the epidermis of the SAM apparently directs local sites of auxin accumulation and these sites correspond to the position at which the organ primordia develop ${ }^{(9,26-28)}$. This process requires the continuous repositioning of PIN 
proteins to create the sequence of sites of auxin accumulation needed to trigger the initiation of successive organs.

Auxin also plays a key role in the development of the vascular network which extends throughout the plant. The vascular bundles of the stem link the intricate patterns of veins in leaves to the central vascular core of the $\operatorname{root}^{(29)}$. Canalisation theory is the most prominent theory for the mode of action of auxin in vascular patterning. Canalisation describes positive feedback between auxin, auxin transport, and its polarisation. This results in the establishment of canals of auxin transporting cell files connecting an auxin source to an auxin sink. These canals later differentiate into vascular tissue.

Developing leaves are strong sources of auxin ${ }^{(30-32)}$. Auxin is transported through the expanding leaves and exported into the main stem, where it is conducted downward in files of cells associated with the vascular bundles. These cells express high levels of basally localised PIN proteins ${ }^{(33)}$. The root of a plant acts as sink for this auxin which is transported into the central vascular cylinder of the root towards the root tip. At the tip, a complex pattern of PIN protein localisation results in the cycling of auxin upwards in the outer cell layers of the root, and then back into the central tissues and down toward the root tip again. This auxin transport loop plays a major role in the patterning of the root apical meristem, defining the zones of cell division, differentiation and elongation ${ }^{(5-6)}$.

The basipetal flux of auxin down the plant from the shoot to the root is called the polar transport stream and provides a systemic source of information about the health and activity of shoot apices ${ }^{(10)}$. One role for this transport stream is in the regulation of shoot branching. Shoot branches arise from secondary SAMs established in the axil of each leaf formed by the primary shoot meristem. These axillary meristems may remain dormant, or they may activate to produce a shoot branch. Auxin, produced by active shoot apices with young expanding leaves, is exported into the stem and has an inhibitory influence on the activity of the axillary meristems below, a phenomenon known as apical dominance ${ }^{(34-35)}$. Removal of the active shoots results in release of the inhibited axillary meristems, which can be prevented by application of auxin to the decapitated stump. The auxin in the polar transport stream inhibits bud growth indirectly, since it does not enter the bud ${ }^{(36-37)}$. Several non-exclusive mechanisms have been proposed to account for this. These include: downregulation of the synthesis the plant hormone cytokinin, which is a promoter of axillary buds activation $^{(38)}$; up-regulation of the synthesis of a third hormone likely to be a strigolactone or related compound that inhibits bud activation ${ }^{(39-40)}$; and prevention of auxin transport canalisation out of the axillary bud by reducing main stem sink strength ${ }^{(41-43)}$.

The examples above illustrate that the auxin transport network is critical for regulating plant development. Particularly striking are the complex positional dynamics of the PIN protein family of auxin transporters. This highlights the question of how PIN protein localisation is regulated. Relatively little is known about this regulation, but it is clear that auxin itself plays a central role. Auxin is known to regulate PINs at multiple stages, affecting the transcription of PIN genes ${ }^{(44)}$, the cycling of PIN proteins between the plasma membrane and endomembrane compartments ${ }^{(45-48)}$, and the stability of PINs ${ }^{(49)}$. Since the amount of auxin both inside and outside cells is affected by PINs, auxins and their transporters are intimately linked in complex feedback loops. The complexities of these feedback loops and the demanding nature of the cell biology and biochemistry required to probe the 
mechanisms of PIN localisation have created a fertile ground for computational modelling to test the plausibility of hypotheses and prioritise wet experiments.

\section{Models of Biological Systems}

Interest in using modelling as a tool for studying biological systems has grown with the increasing understanding of the molecular components from which the systems are built, coupled with the increasing power of computers. When building biological models care must be taken to ensure that enough biological detail is captured without over complicating the models. This is achieved by making well-informed, thoughtful simplifying assumptions, and working at the appropriate level of abstraction.

In the case of auxin transport models, measurement of the change in concentration of the main players over time in order to get accurate parameters for the models is very challenging. Therefore, validation of hypotheses generated by models has been mostly at a more macroscopic level, involving successful reproduction of observable plant systems behaviour in silico (e.g. by reproducing laser ablation of tissue parts in the model ${ }^{(6,26)}$ ).

A particular trend has been to build models based on alternative unsupported assumptions such as postulating a hypothetical sensory mechanism ${ }^{(50-52)}$. If such models are able to reproduce complex plant behaviours then this indicates that the assumptions are plausible and can guide experiments aimed at testing their validity.

In the examples below we focus on the power of this approach in testing alternative hypotheses rather than the extent to which each model has been validated. Other reviews have touched on the need for careful parameter choice and model validation ${ }^{(53)}$.

\section{Static PIN Models}

Even without feedback regulation, the complex patterns of PIN localisation observed in plant tissues make predicting auxin distribution non-intuitive, especially in growing tissues. To help understand these systems, models using patterns of PIN localisation that are obtained from immunocytochemistry of real plant tissues have been developed. These PIN accumulation patterns are used as templates by which cell-to-cell transport of auxin is determined. One application of this approach is to investigate the robustness of auxin transport routes and auxin distributions, such as the stability of an auxin maximum in root tips $^{(6)}$ or the dynamics of auxin accumulation in the SAM ${ }^{(54)}$. Dynamism in PIN allocation in these systems is limited to PIN redistribution caused by cells changing their fate and thus adopting a different PIN accumulation pattern ${ }^{(6)}$, or by limited auxin redistribution such as occurs during gravitropism ${ }^{(55-56)}$.

\section{Venation Patterns and Canalisation Models}

Canalisation is an attractive target for modelling because it is a self-organising process, where feedback between auxin and its transport should result in emergent patterns of auxin transport pathways. As described above, the venation patterns observed in leaves, and their connection with the vascular tissue of the main stem, are hypothesised to be established by canalisation. It is not yet fully understood how this single mechanism is able to generate the wide range of venation patterns observed in nature, such as parallel veins, branching veins and reticulated networks ${ }^{(57)}$. 
Although the first mathematical models of polar auxin transport date back to $1966^{(58)}$, it is the computer models developed by G. J. Mitchison ${ }^{(59-61)}$, informed by the experimental work of Tsvi Sachs ${ }^{(62-64)}$, that are the foundation of canalisation modelling today. Using pea stem segments, Sachs conducted a number of experiments with different arrangements of auxin sources and sinks. He was able to show that vascular strands differentiate in a narrow path connecting auxin sources, such as exogenously applied auxin, to auxin sinks, such as the auxin transporting tissues of existing vascular strands. Mitchison proposed and modelled two possible mechanisms for canalisation: polar transport and facilitated diffusion.

Mitchison's models were executed over a 2D grid of square cells with auxin moving directly from one cell to the next (i.e. no apoplast). This is a common technique used in tissue simulations, where the behaviour of cell membranes and cell walls is simplified into equations that determine the amount of auxin that moves from one cell to its neighbour. In Mitchison's polar transport model, the transporters are recruited to the membrane of a cell in proportion to the flux of auxin crossing that membrane: the greater the flux, the more transporters are recruited, and the more auxin is transported. This positive feedback can result in the formation of files of cells between an auxin source and a sink. More complex patterns, similar to leaf venation patterns, can also be generated, such as the linking of two canalised cell files to form closed loops ${ }^{(59)}$. Fig. 2 shows canalisation of cells towards an auxin sink.

Mitchison's early canalisation models predicted high auxin flux but low concentration in the canals when compared to the surrounding tissue. The biological evidence, acquired through experiments using reporters such as DR5:GFP, suggests that the canals in fact have high auxin concentrations ${ }^{(65)}$. Kramer $^{(50)}$ has shown that canals with high flux and high concentration can be formed with the addition of auxin influx transporters that actively pump auxin into cells in the canal from the surrounding apoplast, or efflux transporters that pump auxin out of cells bordering the canal towards the cells of the canal.

High concentration canals can also be generated by varying the way in which transporter allocation is regulated by auxin flux. Based on Mitchison's work, Feugier ${ }^{(66)}$ simulated canalisation events in a leaf, represented by a grid of hexagonal cells. Canals of high auxin concentration and high flux can be achieved by having the six faces of the cells compete for PINs from a single available pool. This method of PIN allocation is able to produce a branched pattern of veins, but without the closed loops seen in leaves. If the cell faces acquired PINs in proportion to their flux, but independently of each other, the resulting canals showed low auxin concentration (as in Mitchison ${ }^{(59)}$ ). The nature of the relationship between auxin flux through a particular cell membrane and the allocation of PINs to that membrane is also an important factor in the formation of canals in Feugier et al's model ${ }^{(66)}$.

Mitchison's second proposed mechanism for auxin transport is facilitated diffusion. The facilitated diffusion idea is controversial because it is very mechanistically obscure. In this model auxin is able to diffuse between cells in either direction with a diffusion constant that increases with flux. Mitchison was able to show that this mechanism was able to produce an open, branching vein system. More recent modelling of this mechanism has shown that in some cases, a facilitated diffusion model is more efficient than a polar transport model at joining a source of auxin to a sink ${ }^{(67)}$. The two methods were compared on the same layout of $2 \mathrm{D}$ cells, with one auxin source and one sink. The polar transport model produced a vein that initially went past the sink but was able to connect to it later on. However, with source 
and sink in the same position, facilitated diffusion was able to join them along something close to the shortest path.

\section{Modelling Phyllotaxis}

Like canalisation, phyllotaxis is also a self-organising system with dynamic changes in auxin transport at its centre. As such, it has been a strong focus for modelling approaches.

Phyllotaxis occurs in the shoot apical meristem (SAM). The SAM has a domed shape and is divided into two areas. The central zone (CZ) is a small group of undifferentiated stem cells that divide to replace themselves while feeding daughter cells into the surrounding peripheral zone (PZ). In the $\mathrm{PZ}$, cells are able to differentiate into leaf founder cells. Leaf specification apparently occurs in the epidermis (the outermost cell layer), triggered by local auxin accumulation ${ }^{(68)}$. An early event is the formation of the leaf's mid-vein in the underlying tissue, which provides the vascular link between the developing leaf primordium and the existing vasculature in the stem below.

The spacing of successive leaves during phyllotaxis is highly regular. Hofmeister ${ }^{(28)}$ suggested that a new primordium would form as far away as possible from all existing primordia; this led to inhibition models of phyllotaxis, where primordia produce a factor that prevents new primordia forming nearby. Most models of phyllotaxis have followed this basic principle, with either a lack of activator or presence of an inhibitor, or both, ensuring that primordia form with the correct spacing. However, auxin-induced patterning is different from reaction-diffusion models since active transport has to be considered ${ }^{(69)}$. Therefore, with mounting evidence that phyllotaxis is regulated at least in part by PIN-mediated patterns of local auxin accumulation, the majority of phyllotaxis models have focused specifically on auxin and its transport ${ }^{(26,52,70-71)}$.

\section{Up-the-gradient models}

The fact that PIN proteins orient toward the sites of leaf specifications at which auxin accumulates has suggested that a canalisation-type mechanism, in which auxin fluxes direct PIN localisation, may not be able to account for phyllotaxis. An alternative system for PIN polarisation has therefore been suggested in which PIN proteins are preferentially allocated to the membrane facing the neighbouring cell with highest auxin concentration ${ }^{(72)}$ (Fig.3). These models are here referred to as up-the-gradient models ${ }^{(73)}$. Once one cell has a slightly higher auxin concentration than its neighbours, the neighbours preferentially orient their PIN proteins towards it. This creates a positive feedback loop that accumulates auxin in a small number of cells and depletes the surrounding tissue of auxin. Models using this mechanism are able to reproduce the positioning of primordia seen in phyllotaxis on both static and growing 2D tissue grids ${ }^{(52,71)}$. These models depend on the ability of cells to report their auxin concentration to their neighbours, by an as yet unknown mechanism.

If these models are correct, the PINs of the epidermis are positioned based on auxin concentration. However, the formation of the midvein immediately below the site of auxin accumulation involves PIN orientation towards low auxin concentration in a source-to-sink pattern typical of classical with-the-flux canalisation models. This means that either there are at least two distinct mechanisms for positioning PINs, capable of operating in close proximity, or one of these mechanisms does not occur.

\section{Unifying models}

Attempts have been made to develop simulations that can explain both phyllotaxis and canalisation, using only one PIN allocation mechanism. Merks et al. ${ }^{(74)}$ propose the 
travelling-wave hypothesis, an up-the-gradient based concentration-dependent mechanism where a transport canal in a leaf could be formed by PIN polarising towards the neighbouring cell of highest auxin concentration. Modelled on a leaf template, an unstable local auxin maximum develops at the leaf margin. The cells in the maximum align their PIN towards their neighbours of highest concentration, which causes the maximum to move, leaving a trail of polarised cells behind it. This model has yet to be rigorously tested against experimental evidence.

On the other hand, Stoma et al. ${ }^{(73)}$ have shown that it is possible to model phyllotaxis using only flux to control the polarisation of PIN. They show that flux-driven PIN polarisation can occur both down and up an auxin concentration gradient, based on the definition of two types of auxin sinks. Weak sinks lead to auxin maximum formation in the L1, while strong sinks induce the formation of provascular strands in the tissue below. During the simulation, auxin moves towards weak sinks. As soon as auxin concentration in a weak sink cell reaches a critical level, canalisation towards the inner layers of the SAM, a strong sink, is initiated. Above a threshold of auxin concentration, sink cells change their identity from epidermal to leaf primordia cells. This identity parameter allows for a developmental switch between phyllotaxis and canalisation. Interestingly, while both mechanisms are flux-driven, the choice the system makes between two types of fluxes does happen gradually, requiring a binary switch mechanism. The models also predict a transient reduction in auxin concentration at the point of leaf specification. There is currently no evidence to support this.

An alternative approach was proposed by Bayer et al. ${ }^{(51)}$, who developed a hybrid model with both up-the-gradient and with-the-flux PIN polarisation. The two processes are combined in the simulation so that at low concentrations of auxin the polarisation is withthe-flux, but as the auxin concentration increases, the mechanism changes to up-thegradient. This system is able to simulate primordia formation in the SAM epidermis, which once formed can produce a midvein through the inner layer, joining an existing sink - the vasculature of the stem. This model predicts a transient polarisation of PIN in the inner tissue towards the epidermal layer, near the site of midvein formation. This prediction was subsequently observed in both Arabidopsis and Tomato meristems. One difficulty with this model is that although a midvein can form, it reliably connects to the sink only if the sink produces an additional attractive signal to advertise its location. There is currently no evidence for the existence of such a signal, although there is a long-running debate in the literature about the role of the stem vasculature in contributing to phyllotactic patterning in the meristem above ${ }^{(75-76)}$.

\section{Underlying PIN localisation mechanisms}

Much discussion about the validity of different auxin transport models centres on the plausibility of the underlying PIN localisation mechanisms. The simplifying and mechanistic assumptions of a model must strike a balance between remaining faithful to the biology and minimising complexity. Often it is the assumptions that can tell us the most about how a model works and may indicate where we need more biological data.

For example, for many canalisation models auxin flux is a key piece of information required to achieve correct PIN polarisation (Fig.4a). This presents a difficulty as currently there is no known biological mechanism for measuring auxin flux.

Kramer $^{(77)}$ showed that it is possible to use the internal gradient of auxin created by PIN activity to polarise the PIN proteins in the direction of an auxin sink (Fig 4c). In this model 
PIN mediated efflux would cause auxin depletion from cytoplasmic areas around the PINs. This would lead to an intracellular gradient of auxin. If PIN is preferentially delivered to the membrane at the side of the cell with a lower cytoplasmic auxin concentration, this would lead to polarisation towards an already existing flux. Although this model results in PIN allocation proportional to auxin flux, it is also mechanistically obscure because it requires a gradient-sensing molecule in the cytoplasm.

Another possibility for cells to measure flux would depend on tally molecules localised at the membrane ${ }^{(78)}$. As one auxin molecule moves through a cell, it causes a tally molecule to be moved to the site of its efflux. This would lead to an unequal distribution of tally molecules corresponding to the direction of auxin flux.

In up-the-gradient models the localisation of PIN proteins in a cell is hypothesised to occur preferentially towards neighbours with higher auxin concentrations (Fig.4b). Although there are known mechanisms by which overall cellular auxin concentration is sensed, how this information might be transmitted to neighbouring cells is unknown. Two ideas emerging from modelling are that an unknown signalling molecule transfers information about intracellular auxin concentration between cells ${ }^{(52)}$, or that auxin-induced changes in cell expansion generate physical forces that are sensed by neighbours ${ }^{(79-80)}$.

\section{Whole Plant Models}

Whole plant models have the advantage of allowing us to investigate the role of mechanisms like canalisation in a wider context, including integrating aspects of the plant's external environment. Prusinkiewicz et al. ${ }^{(43)}$ hypothesise that the canalisation described by Mitchison ${ }^{(59)}$ can be implemented at the level of entire shoot segments, allowing efficient modelling of the polar transport stream. As described above, one role for this auxin is the inhibition of shoot branching through prevention of the activation of axillary buds formed at the base each leaf. Experiments with radiolabeled auxin have shown that auxin moving down the stem does not enter the axillary buds to inhibit them, but rather the effect is indirect. Prusinkiewicz et al propose that this indirect mechanism is mediated by an auxin transport switch. If canalisation of auxin transport out of the bud is required for bud activation, auxin moving in the stem might reduce its strength as a sink, preventing auxin transport canalisation from the bud.

This model makes an assumption that the many cells of the stem can be approximated as a single segment of auxin transporting tissue. This reduces computation time, and thus allows whole plant level modelling. The model uses a number of these segments to represent the stem. Each stem segment has an attached "bud" which is a potential source of auxin. The nature of the transport switch is demonstrated in a small model of two sources, both connected to a stem segment. The stem is connected to a sink. If either one of the sources is activated auxin begins to flow into the stem - there is a net flux from the source into the stem. This flux increases the PIN on the membrane between the source and stem. Increased PIN causes increased flux, and very quickly positive feedback causes polarisation of PIN towards the stem. If the second source is then activated it now has a high auxin concentration. However, the net flux from source to stem is very low because of high auxin in the stem. The positive feedback on the flux does not occur and the source does not polarise. If both sources are activated in very quick succession, both experience a high net flux in the direction of the stem for long enough for the positive feedback to cause polarisation, and both canalise auxin transport into the stem. 
Using this simple switch mechanism in larger growing plant models, where new segments with "buds" are added at the apex of the plant, the authors were able to reproduce many aspects of plant branching behaviour, including basipetal and acropetal branching patterns and the response to decapitation. Furthermore they were able to model the behaviour of a number of Arabidopsis branching mutants and double mutants affecting auxin transport by changing single model parameters consistent with the known molecular functions of the genes affected by the mutations.

\section{Conclusion and Prospects}

The field of auxin transport modelling has developed rapidly in recent years, reinvigorated by new experimental data. This has allowed for richer models testing richer hypotheses. In particular, both canalisation and phyllotaxis are attractive targets for computer modelling. They are high-level self-organising outcomes of processes occurring within and between cells. One of the goals in the construction of models is to produce emergent processes of interest by modelling what is believed to be going on at the lower levels, avoiding explicitly modelling the emergent process itself. Some models in the field are starting to get closer to this goal. The identification of many more of the biological actors, such as PIN efflux carriers and AUX influx carriers, has greatly helped in this respect.

These developments in model sophistication have benefits, but can also have drawbacks if used without care. Biological data tell us a lot about what is happening but many of the underlying mechanisms are still unknown. PIN polarisation is a good example of this problem. We are able to see it happening but we do not know what is driving it. This is where modelling can help. A model allows us to investigate under what conditions our predictions about the biology work as we expect. Compared with wet experiments, modelling allows for rapid hypothesis testing, and can provide information about whether a hypothesis can achieve what we see in planta, and under what circumstances. If a hypothesis is found that can produce the correct emergent behaviour, then predications from the models can sometimes be used to prioritise experiments for analysis in planta, hopefully leading to a deeper understanding. Yet as our models include ever more biological actors and their interactions, they become ever more complicated. It is important to avoid building models that are difficult to validate or even to understand. As the processing capacity of computers increases this might be an easy trap to fall into, as the natural limit of what is computationally possible shifts. Models should abstract away some complexity to aid understanding; producing a model as complex as a real plant is unhelpful. Also incorporating more biology will lead to more modelling assumptions, both simplifying and mechanistic. It is important to make these modelling assumptions clearly visible as they might provide valuable clues about what to look for in the biology.

The ability of models to prioritise experiments is particularly important in plant cell biology, where the experiments continue to be extremely challenging and basic questions such as the concentration of auxin in the cytoplasm, nucleus and apoplast are still a matter of hot debate. Improvements in methods to measure a wider range of cellular parameters are needed to speed up the model-experiment cycle.

\section{Acknowledgements:}

We would like to thank Lauren Shipley for proof reading the paper. Our auxin transport simulation work is funded by the Biotechnology and Biological Science Research Council of the UK, The Gatsby Foundation, The White Rose University Consortium and Microsoft Research Europe. 


\section{References}

1. Darwin C, Darwin F. The power of movement in plants: London: John Murray; 1880.

2. Friml J, Vieten A, Sauer M, Weijers D, Schwarz H, Hamann T, Offringa R, J rgens G. Efflux-dependent auxin gradients establish the apical-basal axis of Arabidopsis. Nature 2003;426(6963):147-153.

3. Weijers D, Sauer M, Meurette O, Friml Jv, Ljung K, Sandberg G, Hooykaas P, Offringa R. Maintenance of Embryonic Auxin Distribution for Apical-Basal Patterning by PINFORMED-Dependent Auxin Transport in Arabidopsis. The Plant Cell 2005;17(9):25172526.

4. Benjamins R, Scheres B. Auxin: The Looping Star in Plant Development. Annual Review of Plant Biology 2008;59:443-465.

5. Blilou I, Xu J, Wildwater M, Willemsen V, Paponov I, Friml J, Heidstra R, Aida M, Palme K, Scheres B. The PIN auxin efflux facilitator network controls growth and patterning in Arabidopsis roots. Nature 2005;433(7021):39-44.

6. Grieneisen VA, Xu J, Marée AFM, Hogeweg P, Scheres B. Auxin transport is sufficient to generate a maximum and gradient guiding root growth. Nature 2007;449:10081013.

7. Smet ID, Tetsumura T, Rybel BD, Frey NFD, Laplaze L, Casimiro I, Swarup R, Naudts $M$, Vanneste $S$, Audenaert $D$ and others. Auxin-dependent regulation of lateral root positioning in the basal meristem of Arabidopsis. Development 2007;134(4):681690.

8. Kuhlemeier C. Phyllotaxis. Trends in Plant Science 2007;12(4):143-150.

9. Kuhlemeier C, Reinhardt D. Auxin and phyllotaxis. Trends in Plant Science 2001;6(5):187-189.

10. Leyser $\mathrm{O}$. The control of shoot branching: an example of plant information processing. Plant, Cell and Environment 2009;32(6):694-703.

11. Leyser $O$. The fall and rise of apical dominance. Curr Opin Genet Dev 2005;15(4):468-71.

12. Ongaro V, Leyser O. Hormonal control of shoot branching. J Exp Bot 2008;59(1):6774.

13. Sachs T. The control of the patterned differentiation of vascular tissues. Advances in Botanical Research 1981;9:151-262.

14. Ye Z-H, Freshour G, Hahn MG, Burk DH, Zhong R. Vascular development in Arabidopsis. International Review of Cytology 2002;220:225-256.

15. Krupinski $\mathrm{P}$, Jonsson H. Modeling Auxin-regulated Development. Cold Spring Harb Perspect Biol 2010;2(2):a001560.

16. Rubery $\mathrm{PH}$, Sheldrake AR. Carrier-mediated auxin transport. Planta 1974;118(2):101-121.

17. Raven JA. Transport of indoleacetic acid in plant cells in relation to $\mathrm{pH}$ and electrical potential gradients, and its significance for polar IAA transport. New Phytologist $1975 ; 74(2): 163-172$.

18. Geisler M, Murphy AS. The ABC of auxin transport: the role of p-glycoproteins in plant development. FEBS Letters 2006;580(4):1094-1102.

19. Petrálvssek J, Mravec J, Bouchard R, Blakeslee JJ, Abas M, Seifertová D, Wi 'sniewska J, Tadele Z, Kubelvs M, IvCovanová M and others. PIN Proteins Perform a Rate-Limiting Function in Cellular Auxin Efflux. Science 2006;312:914918.

20. Wisniewska J, Xu J, Seifertová D, Brewer PB, Ruzicka K, Blilou I, Rouquié D, Benková $\mathrm{E}$, Scheres B, Friml J. Polar PIN localization directs auxin flow in plants. Science 2006;312(5775):883.

21. Vieten A, Sauer M, Brewer PB, Friml Jv. Molecular and cellular aspects of auxintransport-mediated development. Trends in Plant Science 2007;12(4):1360-1385. 
22. Bandyopadhyay A, Blakeslee JJ, Lee OR, Mravec J, Sauer M, Titapiwatanakun B, Makam SN, Bouchard R, Geisler M, Martinoia E and others. Interactions of PIN and PGP auxin transport mechanisms. Biochemical Sociecty Transactions 2007;35:137141.

23. Dhonukshe P, Tanaka H, Goh T, Ebine K, Mähönen AP, Prasad K, Blilou I, Geldner N, $\mathrm{Xu}$ J, Uemura $\mathrm{T}$ and others. Generation of cell polarity in plants links endocytosis, auxin distribution and cell fate decisions. Nature 2008;456:962-966.

24. Parry G, Marchant A, May S, Swarup R, Swarup K, James N, Graham N, Allen T, Martucci T, Yemm A and others. Quick on the Uptake: Characterization of a Family of Plant Auxin Influx Carriers. Journal of Plant Growth Regulation 2001;20(3):217-225.

25. Friml J, Yang X, Michniewicz M, Weijers D, Quint A, Tietz O, Benjamins R, Ouwerkerk PBF, Ljung K, Sandberg Gr and others. A PINOID-dependent binary switch in apicalbasal PIN polar targeting directs auxin efflux. Science 2004;306(5697):862-865.

26. Reinhardt D, Pesce E-R, Stieger P, Mandel T, Baltensperger K, Bennett M, Traas J, Friml Jv, Kuhlemeier C. Regulation of phyllotaxis by polar auxin transport. Nature 2003;426:255-260.

27. Reinhardt D. Regulation of phyllotaxis. International Journal of Developmental Biology 2005;49:539-546.

28. Hofmeister W. Allgemeine Morphologie der Gewächse. Hofmeister W, editor: Engelmann, Leipzig; 1868. 405-664 p.

29. Scarpella $\mathrm{E}$, Meijer AH. Pattern formation in the vascular system of monocot and dicot plant species. New Phytologist 2004;164:202-242.

30. Ljung K, Bhalerao RP, Sandberg G. Sites and homeostatic control of auxin biosynthesis in Arabidopsis during vegetative growth. Plant Journal 2001;28(4):465474.

31. Zhao Y. The role of local biosynthesis of auxin and cytokinin in plant development. Current Opinion in Plant Biology 2008;11:16-22.

32. Chandler JW. Local auxin production: a small contribution to a big field. BioEssays 2009;31:60-70.

33. Gälweiler L, Guan C, Müller A, Wisman E, Mendgen K, Yephremov A, Palme K. Regulation of polar auxin transport by AtPIN1 in Arabidopsis vascular tissue. Science 1998;282(5397):2226-2230.

34. Thimann KV, Skoog F. Studies on the Growth Hormone of Plants: III. The Inhibiting Action of the Growth Substance on Bud Development. Proceedings of the National Academy of Sciences 1933;19(7):714-716.

35. Cline M. Apical dominance. The Botanical Review 1991;57(4):318-358.

36. Prasad TK, Li X, Abdel-Rahman AM, Hosokawa Z, Cloud NP, Lamotte CE, Cline MG. Does Auxin Play a Role in the Release of Apical Dominance by Shoot Inversion in Ipomoea nil? Annals of Botany 1993;71(3):223-229.

37. Booker J, Chatfield S, Leyser O. Auxin acts in xylem-associated or medullary cells to mediate apical dominance. Plant Cell 2003;15(2):495-507.

38. Tanaka M, Takei K, Kojima M, Sakakibara H, Mori H. Auxin controls local cytokinin biosynthesis in the nodal stem in apical dominance. Plant Journal 2006;45(6):10281036.

39. Umehara M, Hanada A, Yoshida S, Akiyama K, Arite T, Takeda-Kamiya N, Magome H, Kamiya Y, Shirasu K, Yoneyama K and others. Inhibition of shoot branching by new terpenoid plant hormones. Nature 2008;455(7210):195-200.

40. Gomez-Roldan V, Fermas S, Brewer PB, Puech-Pagès V, Dun EA, Pillot J-P, Letisse F, Matusova R, Danoun S, Portais J-C and others. Strigolactone inhibition of shoot branching. Nature 2008;455(7210):189-194.

41. Li C-J, Bangerth F. Autoinhibition of indoleacetic acid transport in the shoots of twobranched pea (Pisum sativum) plants and its relationship to correlative dominance. Physiologia Plantarum 1999;106(4):415-420. 
42. Bennett T, Sieberer T, Willett B, Booker J, Luschnig C, Leyser O. The Arabidopsis MAX pathway controls shoot branching by regulating auxin transport. Current Biology 2006;16(6):553-563.

43. Prusinkiewicz P, Crawford S, Smith RS, Ljung K, Bennett T, Ongaro V, Leyser O. Control of bud activation by an auxin transport switch. Proceedings of the National Academy of Sciences 2009;106(41):17431-17436.

44. Vieten A, Vanneste S, Wisniewska J, Benková E, Benjamins R, Beeckman T, Luschnig C, Friml J. Functional redundancy of PIN proteins is accompanied by auxin-dependent cross-regulation of PIN expression. Development 2005;132(20):4521-4531.

45. Dhonukshe P, Aniento F, Hwang I, Robinson DG, Mravec J, Stierhof Y-D, Friml J. Clathrin-mediated constitutive endocytosis of PIN auxin efflux carriers in Arabidopsis. Current Biology 2007;17(6):520-527.

46. Geldner N, Friml Jv, Stierhof Y-D, Jürgens G, Palme K. Auxin transport inhibitors block PIN1 cycling and vesicle trafficking. Nature 2001;413:425-428.

47. Feraru E, Friml Jv. PIN Polar Targeting. Plant Physiology 2008;147:1553-1559.

48. Kleine-Vehn J, Friml Jv. Polar Targeting and Endocytic Recycling in Auxin-Dependent Plant Development. Annual Review of Cell and Developmental Biology 2008;24:447473.

49. Paciorek T, Zavzímalová E, Ruthardt N, Petrávsek J, Stierhof Y-D, Kleine-Vehn J, Morris DA, Emans N, Jürgens G, Geldner N and others. Auxin inhibits endocytosis and promotes its own efflux from cells. Nature 2005;435:1251-1256.

50. Kramer EM. PIN and AUX/LAX proteins: their role in auxin accumulation. Trends in Plant Science 2004;9(12):578 - 582.

51. Bayer E, Smith R, Mandel T, Nakayama N, Sauer M, Prusinkiewicz P, Kuhlemeier C. Integration of transport-based models for phyllotaxis and midvein formation. Genes and Development 2009;23:373-384.

52. Jönsson $H$, Heisle MG, Shapiro BE, Meyerowitz EM, Mjolsness E. An auxin-driven polarized transport model for phyllotaxis. Proceedings of the National Academy of Sciences 2006;103:1633-1638.

53. Kramer EM. Computer models of auxin transport: a review and commentary. J Exp Bot 2008;59(1):45-53.

54. de Reuille PB, Bohn-Courseau I, Ljung K, Morin H, Carraro N, Godin C, Traas J. Computer simulations reveal properties of the cell-cell signaling network at the shoot apex in Arabidopsis. Proceedings of the National Academy of Sciences 2006;103(5):1627-1632.

55. Young LM, Evans ML, Hertel R. Correlations between gravitropic curvature and auxin movement across gravistimulated roots of Zea mays. Plant Physiology 1990;92:792796.

56. Rashotte AM, Brady SR, Reed RC, Ante SJ, Muday GK. Basipetal auxin transport is required for gravitropism in roots of Arabidopsis. Plant Physiology 2000;122(2):481490.

57. Scarpella E, Marcos D, Friml J, Berleth T. Control of leaf vascular patterning by polar auxin transport. Genes and Development 2006;20(8):1015-1027.

58. Leopold AC, Hall OF. Mathematical model of polar auxin transport. Plant Physiology 1966;41:1476-1480.

59. Mitchison GJ. A model for vein formation in higher plants. Proceedings of the Royal Society B 1980;207:79-109.

60. Mitchison GJ. The Polar Transport of Auxin and Vein Patterns in Plants. Philosophical Transactions of the Royal Society B 1981;295(1978):461-471.

61. Mitchison GJ. The effect of intracellular geometry on auxin transport. Proceedings of the Royal Society London B 1981;214:53-83.

62. Sachs T. The Role of the Root in the Induction of Xylem Differentiation in Peas. Annals of Botany 1968;32(2):391-399. 
63. Sachs T. Polarity and the Induction of Organized Vascular Tissues. Annals of Botany $1969 ; 33(2): 263-275$.

64. Sachs T. Patterned Differentiation in Plants. Differentiation 1978;11(1-3):65-73.

65. Nakamura A, Higuchi K, Goda H, Fujiwara MT, Sawa S, Koshiba T, Shimada Y, Yoshida S. Brassinolide induces IAA5, IAA19, and DR5, a synthetic auxin response element in Arabidopsis, implying a cross talk point of brassinosteroid and auxin signaling. Plant Physiol 2003;133(4):1843-1853.

66. Feugier FG, Mochizuki A, Iwasa Y. Self-organization of the vascular system in plant leaves: inter-dependent dynamics of auxin flux and carrier proteins. Journal of Theoretical Biology 2005;236:366-375.

67. Smith RS, Bayer EM. Auxin transport-feedback models of patterning in plants. Plant, Cell and Environment 2009;32(9):1258-1271.

68. Reinhardt $D$, Mandel $T$, Kuhlemeier $C$. Auxin regulates the initiation and radial position of plant lateral organs. Plant Cell 2000;12(4):507-518.

69. Smith RS. The role of auxin transport in plant patterning mechanisms. PLoS Biol 2008;6(12):e323.

70. Douady S, Couder Y. Phyllotaxis as a physical self-organized growth process. Physical Review Letters 1992;68(13):2098-2101.

71. Smith RS, Guyomarc'h S, Mandel T, Reinhardt D, Kuhlemeier C, Prusinkiewicz P. A plausible model of phyllotaxis. Proceedings of the National Academy of Sciences 2006;103(5):1301-1306.

72. Heisler MG, Ohno C, Das P, Sieber P, Reddy GV, Long JA, Meyerowitz EM. Patterns of auxin transport and gene expression during primordium development revealed by live imaging of the Arabidopsis inflorescence meristem. Curr Biol 2005;15(21):18991911.

73. Stoma S, Lucas M, Chopard J, Schaedel M, Traas J, Godin C. Flux-based transport enhancement as a plausible unifying mechanism for auxin transport in meristem development. PLoS Computational Biology 2008;4(10):e1000207.

74. Merks RMH, Van de Peer Y, Inzé D, Beemster GTS. Canalization without flux sensors: a traveling-wave hypothesis. Trends in Plant Science 2007;12(9):384-390.

75. Larson PR. Development and Organization of the Primary Vascular System in Populus deltoides According to Phyllotaxy. American Journal of Botany 1975;62(10):10841099.

76. Kwiatkowska D. The Relationships between the Primary Vascular System and Phyllotactic Patterns of Anagallis arvensis (Primulaceae). American Journal of Botany $1992 ; 79(8): 904-913$.

77. Kramer EM. Auxin-regulated cell polarity: an inside job? Trends in Plant Science $2009 ; 14(5): 242-247$.

78. Coen E, Rolland-Lagan A-GI, Matthews M, Bangham JA, Prusinkiewicz P. The genetics of geometry. Proceedings of the National Academy of Sciences 2004;101(14):47284735.

79. Green PP. Expression of pattern in plants: combining molecular and calculus-based biophysical paradigms. American Journal of Botany 1999;86(8):1059-1076.

80. Newell AC, Shipman PD, Sun Z. Phyllotaxis: Cooperation and competition between mechanical and biochemical processes. Journal of Theoretical Biology 2008;251:421439. 


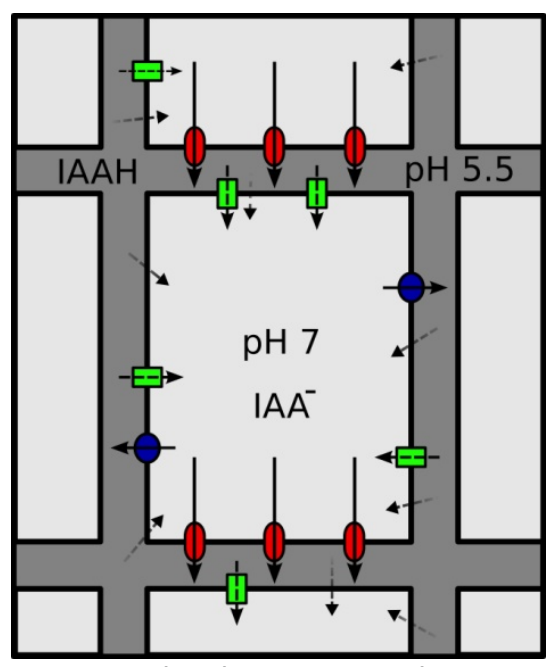

Figure 1. The chemiosmotic theory. Cell wall compartments (dark grey) have a pH around 5.5, while the cytosol is nearly neutral in $\mathrm{pH}$. In the cell wall, part of the auxin is protonated (IAAH), while in the cytosol virtually all auxin appears in its anionic form (IAA-). Arrows indicate the routes of auxin transport over cellular membranes. Auxin in its protonated form is able to enter the cell unaided (bare dotted arrows). Transporter proteins in this figure include importers of the AUX/LAX family (green), and exporters of the PIN (red) and ABCB families (blue).
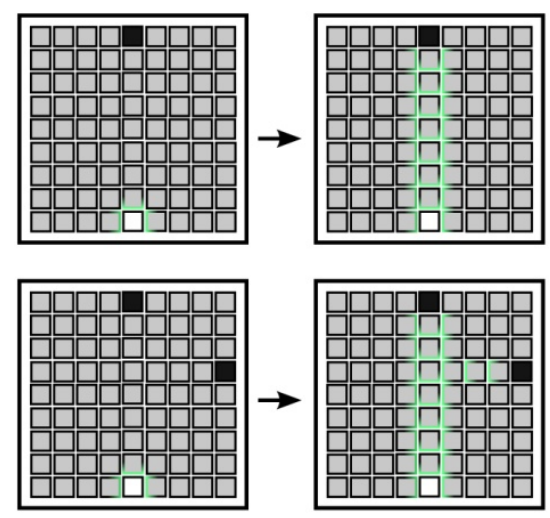

Figure 2: Auxin transport canalisation. A schematic tissue is drawn as a two dimensional grid of square cells. Cells acting as auxin sources are black; auxin sinks are white. PIN localisation in the membrane (green) shows the direction of auxin efflux and thus the direction of overall auxin transport through the tissue. Initial fluxes of auxin (upper left) are reinforced and lead to the development of auxin transport canals between source and sink (upper right). Lower left and lower right: The situation with two source cells. One possible result could be a branching pattern where both canals drain into the same sink. In both situations, auxin is drained from surrounding tissues (grey cells).

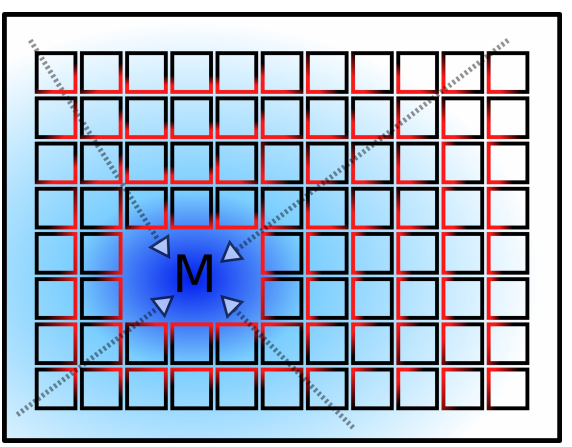

Figure 3: Up-the-gradient transport mechanism of auxin. In phyllotaxy models, auxin is often hypothesised to accumulate at local maxima (M) that precede organ initiation. To illustrate typical PIN polarisation patterns around 
an auxin maximum, the picture shows a schematic tissue of square cells (red: high concentration of PINs in the membrane, black: low PIN concentration, blue: auxin concentration). Arrows represent the direction of auxin transport.

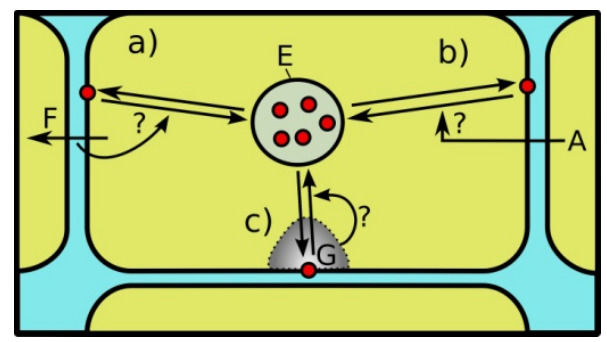

Figure 4. Subcellular events of PIN dynamics, as employed in auxin transport models. a) On the left, the feedback of flux (F) on PIN targeting is shown. PIN proteins (red) cycle between the membrane and endosomal compartments (E). Flux could either influence the insertion or removal of PINs from the membrane. b) The situation in up-the-gradient models: Auxin concentration in the neighbouring cell (A) feeds back on PIN cycling. c) shows the hypothetical feedback on PIN cycling driven by cytosolic auxin gradients $(G)$ around already inserted PINs. The area G shows cytosolic auxin concentration (grey) and lower concentration near PINs (white), where auxin is depleted through active efflux. 